\title{
Zeichen und Wunder
}

Liebe Leserin, lieber Leser,

der Kampf gegen den Klimawandel gleicht der Bekämpfung der Corona-Pandemie: Fachleute empfehlen nachhaltige, systemische Maßnahmen - die Politik setzt sie jedoch oft amateurhaft um. Aber es gibt seit Kurzem Hoffnung: Die EU verlangt den zügigen Einsatz von synthetischen und Biokraftstoffen. In Deutschland stuft Agora Energiewende diese als wichtig zur Erreichung der Klimaziele ein. Selbst ExUmwelt-Staatssekretär Jochen Flasbarth vertritt diese Botschaft vor der Presse. Und auch die neue Bundesregierung setzt sich im Koalitionsvertrag „dafür ein, dass nachweisbar nur mit E-Fuels betankbare Fahrzeuge neu zugelassen werden können“.

Eine Reduktion der globalen $\mathrm{CO}_{2}$-Emissionen des Verkehrssektors ist machbar. Aussicht auf Erfolg hat dies aber nur mit Elektrizität und dem flächendeckenden Einsatz flüssiger oder gasförmiger Energieträger auf nachhaltiger Basis. Aktuell sind in Deutschland 57 Millionen, in der EU rund 250 Millionen und global etwa 1,4 Milliarden Fahrzeuge im Umlauf. Eine Umweltpolitik, die das nicht berücksichtigt, ist sinnlos. Sicher, genug Wind- und Solarstrom ließe sich vielleicht produzieren, aber leider nicht puffern, da bisher keine Anlagen dafür bereitstehen. Und auch die Netzinfrastruktur ist vielerorts nicht dafür ausgelegt, pro Straße mehrere E-Fahrzeuge sicher zu versorgen.

Die Koalitionäre versprechen auch, dass „gemäß den Vorschlägen der Europäischen Kommission [...] im Verkehrsbereich in Europa 2035 nur noch $\mathrm{CO}_{2}$-neutrale Fahrzeuge zugelassen [werden] " und „setzen [sich] für die Verabschiedung einer [...] umsetzbaren Schadstoffnorm Euro 7 ein“. Dies soll „technologieoffen“ ausgestaltet werden. Und hier kommen E-Fuels und Biokraftstoffe ins Spiel: Mit synthetischen Kraftstoffen betriebene, thermodynamische Energiewand- ler wirken in der Gesamtbilanz ebenso defossilisierend wie rein elektrische Antriebe. So beträgt der Treibhausgaseintrag über den gesamten Lebenszyklus mit heutigen Energieträgern $31 \mathrm{t} \mathrm{CO}_{2}$ bei einem Pkw mit Verbrennungsmotor, $24 \mathrm{t}$ bei einem BEV und $32 \mathrm{t}$ bei einem FCEV. Kommen synthetische Kraftstoffe zum Einsatz, liegen die drei Antriebsformen nahezu gleichauf.

Nötig ist eine systemische und technologieoffene Betrachtung von Energieträgern und -wandlern über die gesamte Wirkungskette. So lassen sich die Potenziale für eine nachhaltige globale $\mathrm{CO}_{2}{ }^{-}$ Senkung heben. Die Aufgabe von Wissenschaft, Politik, Industrie und Medien ist es, die Diskussion als ein „Sowohl-als-auch“ in Richtung Next Generation Engines in einem nachhaltigen Energiesystem zu gestalten. Es gibt nun Zeichen aus der Politik, die wieder Hoffnung machen.

PS: Ab sofort erscheint der Hansen-Report on Automotive Electronics im englischen Original - der Authentizität wegen.

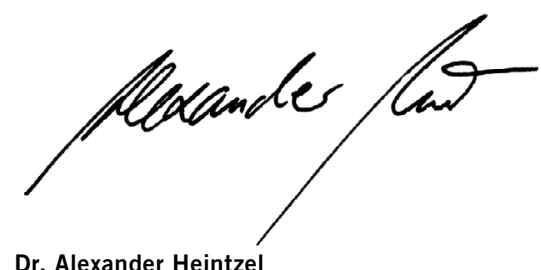

Chefredakteur

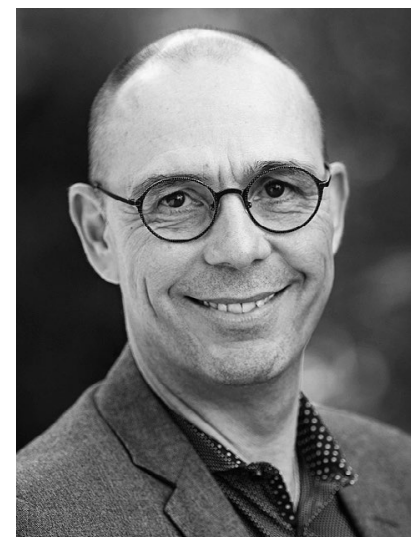

